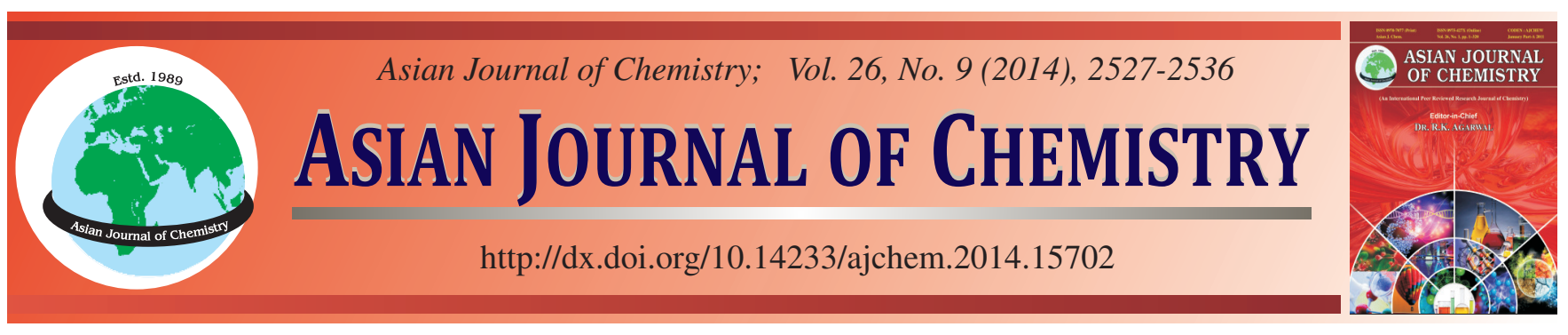

\title{
Evaluation of Chemical and Physico-Chemical Indicators of Water in the Lakes of Barlinek-Gorzów Landscape Park (North-West Poland)
}

\section{PIOTR DANISZEWSKI}

Department of Invertebrate Zoology and Limnology, Faculty of Biology University of Szczecin, 13 Waska Street, 71-415 Szczecin, Poland Corresponding author: E-mail: daniszewski73@gmail.com

\begin{abstract}
This paper presents the evaluation of physico-chemical parameters of lake water in the Barlinek-Gorzów Landscape Park based on the European Union Water Framework Directive.The research was carried out in the years 2008-2012, between April and October. From each of the three measuring stations located in the lakes included the study, two separate water samples were taken for chemical analysis. Upon sampling, the water $\mathrm{pH}$ was measured. Water was tested in compliance with the Polish Standards. Collected water samples were stabilized pursuant to the guidelines of the Polish Standards. Other indicators of water quality were marked within $24 \mathrm{~h}$ of sampling. The oxidation of dissolved organic matter was measured with the COD-Mn method, in accordance with Polish Standards. The $\mathrm{pH}$ values in the neutral range-7.48 to 7.85 in all lakes water. According to the classification of the European Union Water Framework Directive, all lakes were classified as first class. The tests have demonstrated that water quality in the lakes with regard to the tested indicators varied. By analyzing the average annual values, one can note that the $\mathrm{pH}, \mathrm{O}_{2 \text { diss. }}$ and $\mathrm{NO}_{3}{ }^{-}$concentration showed a relatively small variation in all the investigated lakes. The total suspended solids (TSS) in the lakes Barlineckie, Glebokie, Suche, Lubiszewko, Przyleg and Chlop, fell into the II class, while the lakes Lubie and Wielgie met the criteria of the III class. The $\mathrm{P}_{\text {total }}$ concentrations in the surface layer of the lakes was little differentiated, reaching the levels appropriate for the II and III quality class according to the classification of the European Union Water Framework Directive. The total phosphorus concentration was $0.21-0.67 \mathrm{mg} \mathrm{dm}^{-3}$. The highest concentration of total phosphorus was recorded in Lakes Przyleg and Wielgie. The total suspended solids in the lakes Barlineckie, Glebokie, Suche, Lubiszewko, Przyleg and Chlop, fell into the II class, while the lakes Lubie and Wielgie met the criteria of the III class. The $\mathrm{P}_{\text {total }}$ concentrations in the surface layer of the lakes was little differentiated, reaching the levels appropriate for the II and III quality class according to the classification of the European Union Water Framework Directive.
\end{abstract}

Keywords: Water, Lake, Barlinek-Gorzów Landscape Park, European Union Water Framework Directive.

\section{INTRODUCTION}

Urbanization is the cause of many changes which are taking place in the environment, including those found in the catchment ${ }^{1-8}$. With this in mind, it is an important issue to properly protect water reservoirs and also take action to counter the adverse effects of human activities on the natural environment, including water bodies ${ }^{2,9-15}$. To address the increasing degradation of surface waters in the European Union, the approach to the evaluation and protection of water resources was changed. This approach was formulated in the European Union Water Framework Directive (2000/60/EC), which calls for the protection of water, as well as an environment-friendly and comprehensive approach to water assessment $t^{2-6,16-21}$. The ecological status of surface waters and groundwater is assessed on the basis of the ecological potential of the biological and physico-chemical and hydromorphological indicators, ${ }^{410,12,17,22-27}$. The goal of the Water Framework Directive is to achieve good water status in all the Member States of the European Union $^{1,3,5,7,9,18,22,24,26}$.

This paper presents the evaluation of physico-chemical parameters of lake water in the Barlinek-Gorzów Landscape Park based on the European Union Water Framework Directive.

\section{EXPERIMENTAL}

The Barlinek-Gorzów Landscape Park was established in October 1991. The Barlinek-Gorzów Landscape Park includes more than 55000 ha of forests, lakes, fields, meadows and is characterized by a great diversity of habitats and abundant life forms ${ }^{28}$. To protect the most valuable plant communities and animal habitats, five nature reserves were created within the boundaries of the Park: 
1. Skalisty Jar Libberta includes Libbert's Gorge and the surrounding moraine hills and glacial erratics. It is the only site featuring lime stones and boulders in Western Pomerania and is surrounded by oak and beech forests ${ }^{28}$.

2. "Debina" forest conservation complex known as the Central European wet-ground forest, featuring stately oaks and beeches with some lime, hornbeam and old pine trees. In its clean environment, as many as 50 species of arboreal lichen have been preserved ${ }^{28}$.

3. Markowe Blota - marshland, with its typical vegetation such as the Sphagnopsida, wild rosemary, ordinary cranberry, cottongrass. The site is visited quite often by white-tailed eagles ${ }^{28}$.

4. The water reserve of the "River Przylezek". It includes a section of the River, the slopes of the riverbank and the surrounding beech stand with some tree specimens that are more than 100 years old. Seen as the watercourse resembles mountain streams, with its pure and cold water, it provides appropriate conditions for Salmonidae to live and spawn ${ }^{28}$.

5. The forest reserve "Wilanów" aims to protects the natural mixed forest with vintage beech, oak and pine trees. Thanks to the varied topography, diverse rare types of forests have been preserved here ${ }^{28}$.

Nature conservation in the park also includes natural monuments, animate and inanimate: 41 trees, 1 boulder, 3 rocks and the natural spring Bozy Dar ${ }^{28}$.

The study covered seven lakes within the boundaries of the Barlinek-Gorzów Landscape Park: Barlineckie Lake, Glebokie Lake, Lubiszewko Lake, Przyleg Lake, Chlop Lake, Lubie Lake, Wielgie Lake.

Barlineckie Lake: The area of the Lake covers 260 hectares, the depth reaches $18 \mathrm{~m}$, max length is $3.8 \mathrm{~km}$ Lake is located in the North. Barlineckie parts of the Park, at a height of $57 \mathrm{~m}$ above sea level and is part of the Mysliborskie ${ }^{28}$.

Glebokie Lake: The surface of the Lake Glebokie in Barlinek is: 4.65 ha, maximum depth $-8 \mathrm{~m}^{28}$.

Lubiszewko Lake: The surface of this Lake covers 52 ha, depth, width is $11.8 \mathrm{~m}$ dating back to $520 \mathrm{~m}$, length up to 2100 $\mathrm{m}$. Lake is located at a height of $63.3 \mathrm{~m}$ above sea level ${ }^{28}$.

Przyleg Lake: The surface of the Lake is 43.2 ha, depth to $5.9 \mathrm{~m}, 650.0 \mathrm{~m}$ width, the length of $1,100 \mathrm{~m}^{28}$.

Chlop Lake: The surface of the Lake is 58.7 ha, depth goes back to $16.0 \mathrm{~m}$, width of $470 \mathrm{~m}$ and length to $1760 \mathrm{~m}$. Lake is located on the height of $59.1 \mathrm{~m}$ above sea level and belongs to the catchment area of the River Santocznej ${ }^{28}$.

Lubie Lake: The surface of the Lake is 58.7 ha, depth goes back to $16.0 \mathrm{~m}$, width of $470 \mathrm{~m}$ and length to $1760 \mathrm{~m}$. Lake is located on the height of the peasant $59.1 \mathrm{~m}$ above sea level and belongs to the catchment area of the river Santoczna ${ }^{28}$.

Wielgie (Dankowskie) Lake: The area of the Lake covers 90.2 ha, depth is $7.7 \mathrm{~m}$, width $920 \mathrm{~m}$ and the length of the 1880 $\mathrm{m}$. It is situated at a height of 69.7 metres above sea level, the Lake is located in the western Part of the Dobiegniewskie ${ }^{28}$.

The research was carried out in the years 2008-2012, between April and October. From each of the three measuring stations located in the lakes included the study, two separate water samples were taken for chemical analysis. Upon sampling, the water $\mathrm{pH}$ was measured. Water was tested in compliance with the Polish Standards. Collected water samples were stabi- lized pursuant to the guidelines of the Polish Standards ${ }^{1-5,11}$. Other indicators of water quality were marked within $24 \mathrm{~h}$ of sampling. The oxidation of dissolved organic matter was measured with the COD-Mn method, in accordance with Polish Standards ${ }^{1-5,11}$. Dissolved oxygen was marked in accordance with the methodology described by Winkler in Daniszewski's work $^{1-4,11}$

The degree of water oxygenation was specified by arrays described by Nemerow ${ }^{7}$. The levels of Total Suspended Solids, $\mathrm{BOD}_{5}, \mathrm{NH}_{4}{ }^{+}, \mathrm{NO}_{2}{ }^{-}, \mathrm{NO}_{3}{ }^{-}, \mathrm{PO}_{4}{ }^{3-}$ diss and $\mathrm{P}_{\text {tot }}$. were marked-in accordance with the methodology described by Daniszewski ${ }^{1-5,11}$. The quality objectives were evaluated according to the criteria recommended for assessing inland surface waters as set out in the European Union Water Framework Directive (Directive 2000/60/EC).

\section{RESULTS AND DISCUSSION}

The results for the seven lakes of the Barlinek-Gorzów Landscape Park, along with the classification in accordance with the European Union Water Framework Directive are presented in Tables-1-7. The $\mathrm{pH}$ of the water in the lakes is influenced by the physico-chemical and biotic interactions of environmental factors ${ }^{1-5,9,12,-14,16,18}$.

Among others, the degree of acidity directly affects life processes occurring in ecosystems. It is responsible for the correct uptake of nutrients by organisms. High alkalinity is beneficial for assimilation and therefore, the nitrogen and phosphorus compounds found in water are much more accessible than in an acid medium. Apart from high acidity, excessive alkalinity of natural waters ( $\mathrm{pH}$ above 9) also has a clearly detrimental impact on organisms $s^{5,12-14,18,24-27,29}$. The studied lakes had $\mathrm{pH}$ values in the neutral range from 7.48 to 7.85 . According to the classification of the European Union Water Framework Directive, all lakes were classified as first class.

The aquatic ecosystems of the studied lakes experienced loss on ignition and non-corresponding values of COD-Mn according to the estimates, which were based on the measurements of "loss on drying" and "residue on ignition" in accordance with the methodology set out by Trojanowski et al. ${ }^{30}$ and on the basis of COD-Mn results, which invariably matched III class water quality. In the lake waters tested, considerable levels of organic matter, including reducing agents, were maintained throughout the year. The reasons for this state of affairs should also be sought in the lake bed sediment, which is rich in organic matter ${ }^{1-5,7,11,12,14,15,18,20-27,29,30}$. The most important elements involved in primary production are phosphorus and nitrogen $^{1-5,7,11,12,14,15,18,20-27,29,30}$. The presence of these substances determines the productivity of a water body, as well as its quality. One nutrient significantly affecting the quality of water is phosphorus ${ }^{1-5,7,11,12,14,15,18,20-25,29,30}$. It is the primary factor which constrains the development of phytoplankton and thus affects massive algal blooms. It can occur in water bodies in the form of inorganic phosphorus as well as dissolved organic forms ${ }^{1-5,7,11,12,14,15,18,20-23}$. Phosphates, or the mineral forms of phosphorus, are best absorbed by organisms and play a huge role in the primary production of a reservoir ${ }^{20,23}$. They are involved in the circulation of matter in any water body. Therefore, one should pay attention to phosphorus compounds in the demersal zone $e^{1-5,7,9-12,14,15,18,20-25,29,30}$. Nitrogen occurs in 


\begin{tabular}{|c|c|c|c|c|c|}
\hline \multicolumn{6}{|c|}{$\begin{array}{c}\text { RESULTS OF THE QUALITY OF SURFACE WATER OF LAKE BARLINECKIE (SPRING, SUMMER AND AUTUMN 2008 - 2012) } \\
\text { ALONG WITH THE CLASSIFICATION VALUES OF INDICATORS ACCORDING TO THE CRITERIA } \\
\text { OF THE EUROPEAN UNION WATER FRAMEWORK DIRECTIVE (2000/60/EC) }\end{array}$} \\
\hline \multicolumn{6}{|c|}{ Year 2008} \\
\hline S.No. & Water quality indices & Units & $\begin{array}{c}\text { 17th April } 2008 \\
\text { Spring }\end{array}$ & $\begin{array}{l}\text { 24th July } \\
\text { Summer }\end{array}$ & $\begin{array}{c}\text { 15th October } 2008 \\
\text { Autumn }\end{array}$ \\
\hline 1. & General suspension & $\mathrm{mg} \mathrm{O}_{2} \mathrm{dm}^{-3}$ & 17.3 (II) & 23.6 (II) & 21.7 (II) \\
\hline 2. & $\mathrm{pH}$ & - & $7.68(\mathrm{I})$ & $7.75(\mathrm{I})$ & $7.80(\mathrm{I})$ \\
\hline 3. & COD-Mn & $\mathrm{mg} \mathrm{O}_{2} \mathrm{dm}^{-3}$ & 7.6 (III) & 9.3 (III) & 8.8 (III) \\
\hline 4. & $\mathrm{BOD}_{5}$ & $\mathrm{mg} \mathrm{O}_{2} \mathrm{dm}^{-3}$ & 3.7 (III) & 5.7 (III) & 4.9 (III) \\
\hline 5. & $\mathrm{O}_{2 \text { diss. }}$ & $\mathrm{mg} \mathrm{O}_{2} \mathrm{dm}^{-3}$ & 8.7 (I) & 9.7 (I) & $7.6(\mathrm{I})$ \\
\hline 6. & $\mathrm{NO}_{3}^{-}$ & $\mathrm{mg} \mathrm{N} \mathrm{dm}{ }^{-3}$ & $0.12(\mathrm{I})$ & $0.28(\mathrm{I})$ & $0.22(\mathrm{I})$ \\
\hline 7. & $\mathrm{NO}_{2}^{-}$ & $\mathrm{mg} \mathrm{N} \mathrm{dm}{ }^{-3}$ & $0.018(\mathrm{I})$ & 0.038 (II) & 0.032 (II) \\
\hline 8. & $\mathrm{NH}_{4}^{+}$ & $\mathrm{mg} \mathrm{N} \mathrm{dm}{ }^{-3}$ & 1.81 (III) & 0.57 (II) & $0.38(\mathrm{I})$ \\
\hline 9. & $\mathrm{PO}_{4}{ }^{3-}$ diss. & $\mathrm{mg} \mathrm{PO} \mathrm{dm}^{-3}$ & $1.03(\mathrm{~V})$ & 0.74 (IV) & 0.42 (III) \\
\hline 10 & $\mathrm{P}_{\text {total }}$ & $\mathrm{mg} \mathrm{P} \mathrm{dm}{ }^{-3}$ & 0.42 (III) & 0.23 (II) & 0.29 (II) \\
\hline \multicolumn{6}{|c|}{ Year 2009} \\
\hline S.No. & Water quality indices & Units & $\begin{array}{c}\text { 15th April } 2009 \\
\text { Spring }\end{array}$ & $\begin{array}{l}\text { 22nd July } 2009 \\
\text { Summer }\end{array}$ & $\begin{array}{l}\text { 21st October } 2009 \\
\text { Autumn }\end{array}$ \\
\hline 1. & General suspension & $\mathrm{mg} \mathrm{O}_{2} \mathrm{dm}^{-3}$ & $18.5(\mathrm{II})$ & 19.7 (II) & $18.0(\mathrm{II})$ \\
\hline 2. & $\mathrm{pH}$ & - & $7.71(\mathrm{I})$ & $7.75(\mathrm{I})$ & $7.75(\mathrm{I})$ \\
\hline 3. & COD-Mn & $\mathrm{mg} \mathrm{O}_{2} \mathrm{dm}^{-3}$ & 7.3 (III) & 8.5 (III) & 8.2 (III) \\
\hline 4. & $\mathrm{BOD}_{5}$ & $\mathrm{mg} \mathrm{O}_{2} \mathrm{dm}^{-3}$ & 4.2 (III) & 4.6 (III) & 3.8 (III) \\
\hline 5. & $\mathrm{O}_{2 \text { diss. }}$ & $\mathrm{mg} \mathrm{O}_{2} \mathrm{dm}^{-3}$ & $7.5(\mathrm{I})$ & $8.6(\mathrm{I})$ & $8.2(\mathrm{I})$ \\
\hline 6. & $\mathrm{NO}_{3}^{-}$ & $\mathrm{mg} \mathrm{N} \mathrm{dm}{ }^{-3}$ & $0.31(\mathrm{I})$ & $0.42(\mathrm{I})$ & 0.28 (I) \\
\hline 7. & $\mathrm{NO}_{2}^{-}$ & $\mathrm{mg} \mathrm{N} \mathrm{dm}-3$ & 0.034 (II) & 0.040 (II) & 0.036 (II) \\
\hline 8. & $\mathrm{NH}_{4}^{+}$ & $\mathrm{mg} \mathrm{N} \mathrm{dm}{ }^{-3}$ & 1.20 (III) & 0.86 (II) & 0.62 (II) \\
\hline 9. & $\mathrm{PO}_{4}^{3-}$ diss. & $\mathrm{mg} \mathrm{PO}_{4} \mathrm{dm}^{-3}$ & 0.68 (III) & 0.53 (III) & 0.47 (III) \\
\hline 10 & $\mathrm{P}_{\text {total }}$ & $\mathrm{mg} \mathrm{P} \mathrm{dm}{ }^{-3}$ & 0.32 (II) & 0.26 (II) & 0.36 (II) \\
\hline \multicolumn{6}{|c|}{ Year 2010} \\
\hline S.No. & Water quality indices & Units & $\begin{array}{c}\text { 21st April } 2010 \\
\text { Spring }\end{array}$ & $\begin{array}{c}\text { 14th July } 2010 \\
\text { Summer }\end{array}$ & $\begin{array}{c}\text { 20th October } 2010 \\
\text { Autumn }\end{array}$ \\
\hline 1. & General suspension & $\mathrm{mg} \mathrm{O}_{2} \mathrm{dm}^{-3}$ & 18.4 (II) & 23.9 (II) & $21.2(\mathrm{II})$ \\
\hline 2. & $\mathrm{pH}$ & - & $7.72(\mathrm{I})$ & $7.70(\mathrm{I})$ & 7.81 (I) \\
\hline 3. & COD-Mn & $\mathrm{mg} \mathrm{O}_{2} \mathrm{dm}^{-3}$ & 6.8 (III) & 7.3 (III) & 7.8 (III) \\
\hline 4. & $\mathrm{BOD}_{5}$ & $\mathrm{mg} \mathrm{O}_{2} \mathrm{dm}^{-3}$ & 4.6 (III) & 5.2 (III) & 4.5 (III) \\
\hline 5. & $\mathrm{O}_{2 \text { diss. }}$ & $\mathrm{mg} \mathrm{O}_{2} \mathrm{dm}^{-3}$ & $7.3(\mathrm{I})$ & 7.9 (I) & 8.1 (I) \\
\hline 6. & $\mathrm{NO}_{3}^{-}$ & $\mathrm{mg} \mathrm{N} \mathrm{dm}{ }^{-3}$ & $0.36(\mathrm{I})$ & 0.29 (I) & 0.48 (I) \\
\hline 7. & $\mathrm{NO}_{2}^{-}$ & $\mathrm{mg} \mathrm{N} \mathrm{dm}-3$ & 0.044 (II) & 0.025 (II) & 0.036 (II) \\
\hline 8. & $\mathrm{NH}_{4}^{+}$ & $\mathrm{mg} \mathrm{N} \mathrm{dm}{ }^{-3}$ & 0.76 (II) & 0.83 (II) & 0.80 (II) \\
\hline 9. & $\mathrm{PO}_{4}^{3-}$ diss. & $\mathrm{mg} \mathrm{PO}_{4} \mathrm{dm}^{-3}$ & 0.52 (III) & 0.44 (III) & 0.65 (III) \\
\hline 10 & $\mathrm{P}_{\text {total }}$ & $\mathrm{mg} \mathrm{P} \mathrm{dm}^{-3}$ & 0.22 (II) & 0.28 (II) & 0.24 (II) \\
\hline \multicolumn{6}{|c|}{ Year 2011} \\
\hline S.No. & Water quality indices & Units & $\begin{array}{l}\text { 20th April } 2011 \\
\text { Spring }\end{array}$ & $\begin{array}{l}\text { 20th July } 2011 \\
\text { Summer }\end{array}$ & $\begin{array}{c}\text { 19th October } 2011 \\
\text { Autumn }\end{array}$ \\
\hline 1. & General suspension & $\mathrm{mg} \mathrm{O}_{2} \mathrm{dm}^{-3}$ & 20.4 (II) & 22.5 (II) & 21.2 (II) \\
\hline 2. & $\mathrm{pH}$ & - & $7.73(\mathrm{I})$ & 7.69 (I) & 7.75 (I) \\
\hline 3. & COD-Mn & $\mathrm{mg} \mathrm{O}_{2} \mathrm{dm}^{-3}$ & 7.2 (III) & 8.5 (III) & 6.7 (III) \\
\hline 4. & $\mathrm{BOD}_{5}$ & $\mathrm{mg} \mathrm{O}_{2} \mathrm{dm}^{-3}$ & 4.7 (III) & 4.2 (III) & 4.6 (III) \\
\hline 5. & $\mathrm{O}_{2 \text { diss. }}$ & $\mathrm{mg} \mathrm{O}_{2} \mathrm{dm}^{-3}$ & 8.2 (I) & $9.0(\mathrm{I})$ & $7.6(\mathrm{I})$ \\
\hline 6. & $\mathrm{NO}_{3}^{-}$ & $\mathrm{mg} \mathrm{N} \mathrm{dm}{ }^{-3}$ & $0.41(\mathrm{I})$ & $0.26(\mathrm{I})$ & 0.21 (I) \\
\hline 7. & $\mathrm{NO}_{2}^{-}$ & $\mathrm{mg} \mathrm{N} \mathrm{dm}^{-3}$ & 0.034 (II) & 0.022 (II) & 0.047 (II) \\
\hline 8. & $\mathrm{NH}_{4}^{+}$ & $\mathrm{mg} \mathrm{N} \mathrm{dm}{ }^{-3}$ & 0.91 (II) & 0.76 (II) & 0.83 (II) \\
\hline 9. & $\mathrm{PO}_{4}^{3-}$ diss. & $\mathrm{mg} \mathrm{PO}_{4} \mathrm{dm}^{-3}$ & 0.36 (II) & 0.41 (III) & 0.47 (III) \\
\hline 10 & $\mathrm{P}_{\text {total }}$ & $\mathrm{mg} \mathrm{P} \mathrm{dm}{ }^{-3}$ & 0.26 (II) & 0.23 (II) & 0.34 (II) \\
\hline \multicolumn{6}{|c|}{ Year 2012} \\
\hline S.No. & Water quality indices & Units & $\begin{array}{c}\text { 18th April } 2012 \\
\text { Spring }\end{array}$ & $\begin{array}{c}\text { 18th July } 2012 \\
\text { Summer }\end{array}$ & $\begin{array}{c}\text { 27th September } 2012 \\
\text { Autumn }\end{array}$ \\
\hline 1. & General suspension & $\mathrm{mg} \mathrm{O}_{2} \mathrm{dm}^{-3}$ & 24.1 (II) & 24.3 (II) & 18.9 (II) \\
\hline 2. & $\mathrm{pH}$ & - & 7.70 (I) & 7.78 (I) & 7.74 (I) \\
\hline 3. & COD-Mn & $\mathrm{mg} \mathrm{O}_{2} \mathrm{dm}^{-3}$ & 8.7 (III) & 9.1 (III) & 7.9 (III) \\
\hline 4. & $\mathrm{BOD}_{5}$ & $\mathrm{mg} \mathrm{O}_{2} \mathrm{dm}^{-3}$ & 3.2 (III) & 5.3 (III) & 4.1 (III) \\
\hline 5. & $\mathrm{O}_{2 \text { diss. }}$ & $\mathrm{mg} \mathrm{O}_{2} \mathrm{dm}^{-3}$ & 7.9 (I) & $8.6(\mathrm{I})$ & 8.1 (I) \\
\hline 6. & $\mathrm{NO}_{3}{ }_{-}^{-}$ & $\mathrm{mg} \mathrm{N} \mathrm{dm}^{-3}$ & $0.38(\mathrm{I})$ & 0.61 (I) & 0.34 (I) \\
\hline 7. & $\mathrm{NO}_{2}^{-}$ & $\mathrm{mg} \mathrm{N} \mathrm{dm}{ }^{-3}$ & 0.039 (II) & 0.032 (II) & 0.035 (II) \\
\hline 8. & $\mathrm{NH}_{4}^{+}$ & $\mathrm{mg} \mathrm{N} \mathrm{dm}{ }^{-3}$ & 0.72 (II) & 0.63 (II) & 0.69 (II) \\
\hline 9. & $\mathrm{PO}_{4}^{3-}$ diss. & $\mathrm{mg} \mathrm{PO}_{4} \mathrm{dm}^{-3}$ & 0.45 (III) & 0.32 (III) & 0.37 (II) \\
\hline 10 & $\mathrm{P}_{\text {total }}$ & $\mathrm{mg} \mathrm{P} \mathrm{dm}{ }^{-3}$ & 0.37 (II) & 0.30 (II) & 0.27 (II) \\
\hline
\end{tabular}




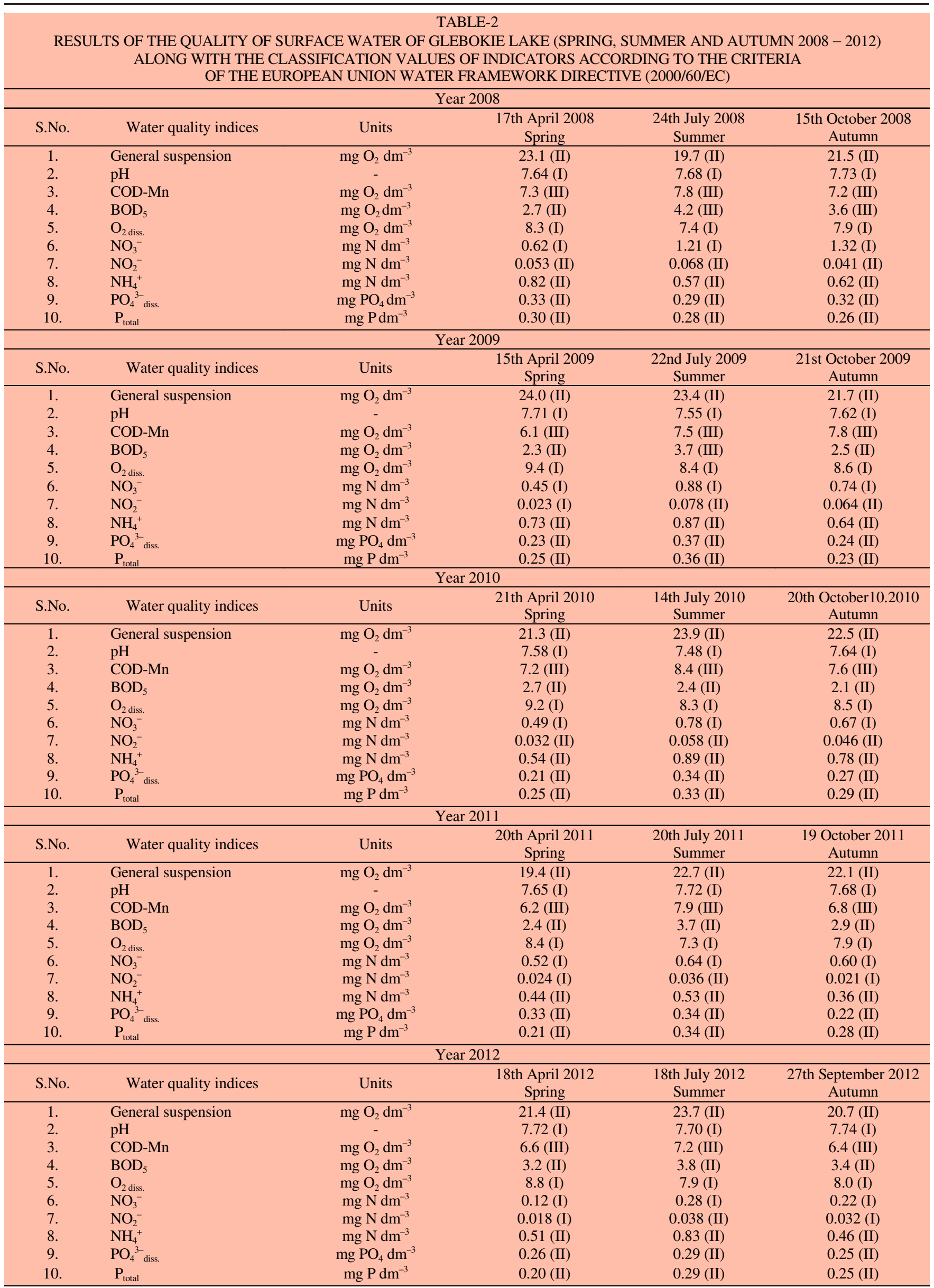




\begin{tabular}{|c|c|c|c|c|c|}
\hline \multicolumn{6}{|c|}{$\begin{array}{l}\text { TABLE-3 } \\
\text { RESULTS OF THE QUALITY OF SURFACE WATER OF LUBISZEWKO LAKE (SPRING, SUMMER AND AUTUMN 2008 - 2012) } \\
\text { ALONG WITH THE CLASSIFICATION VALUES OF INDICATORS ACCORDING TO THE CRITERIA OF } \\
\text { THE EUROPEAN UNION WATER FRAMEWORK DIRECTIVE (2000/60/EC) }\end{array}$} \\
\hline \multicolumn{6}{|c|}{ Year 2008} \\
\hline S.No. & Water quality indices & Units & $\begin{array}{c}\text { 17th April } 2008 \\
\text { Spring }\end{array}$ & $\begin{array}{l}\text { 24th July } 2008 \\
\text { Summer }\end{array}$ & $\begin{array}{c}\text { 15th October } 2008 \\
\text { Autumn }\end{array}$ \\
\hline 1. & General suspension & $\mathrm{mg} \mathrm{O}_{2} \mathrm{dm}^{-3}$ & 16.2 (II) & 19.5 (II) & 18.9 (II) \\
\hline 2. & $\mathrm{pH}$ & - & $7.63(\mathrm{I})$ & $7.72(\mathrm{I})$ & $7.62(\mathrm{I})$ \\
\hline 3. & COD-Mn & $\mathrm{mg} \mathrm{O}_{2} \mathrm{dm}^{-3}$ & 5.3 (II) & 5.7 (II) & 4.8 (II) \\
\hline 4. & $\mathrm{BOD}_{5}$ & $\mathrm{mg} \mathrm{O}_{2} \mathrm{dm}^{-3}$ & 2.1 (II) & 2.5 (II) & 2.2 (II) \\
\hline 5. & $\mathrm{O}_{2 \text { diss. }}$ & $\mathrm{mg} \mathrm{O}_{2} \mathrm{dm}^{-3}$ & $8.4(\mathrm{I})$ & 7.5 (I) & $8.6(\mathrm{I})$ \\
\hline 6. & $\mathrm{NO}_{3}^{-}$ & $\mathrm{mg} \mathrm{N} \mathrm{dm}{ }^{-3}$ & $0.83(\mathrm{I})$ & $1.78(\mathrm{I})$ & $0.92(\mathrm{I})$ \\
\hline 7. & $\mathrm{NO}_{2}^{-}$ & $\mathrm{mg} \mathrm{N} \mathrm{dm}{ }^{-3}$ & 0.053 (I) & 0.085 (II) & 0.069 (II) \\
\hline 8. & $\mathrm{NH}_{4}^{+}$ & $\mathrm{mg} \mathrm{N} \mathrm{dm}{ }^{-3}$ & 0.51 (II) & 0.84 (II) & 0.58 (II) \\
\hline 9. & $\mathrm{PO}_{4}^{3-}$ diss. & $\mathrm{mg} \mathrm{PO}_{4} \mathrm{dm}^{-3}$ & 0.27 (II) & 0.34 (II) & 0.31 (II) \\
\hline 10. & $\mathrm{P}_{\text {Total }}$ & $\mathrm{mg} \mathrm{P} \mathrm{dm}^{-3}$ & 0.21 (II) & 0.33 (II) & 0.25 (II) \\
\hline \multicolumn{6}{|c|}{ Year 2009} \\
\hline S.No. & Water quality indices & Units & $\begin{array}{c}\text { 15th April } 2009 \\
\text { Spring } \\
\end{array}$ & $\begin{array}{c}\text { 22nd July } 2009 \\
\text { Summer }\end{array}$ & $\begin{array}{c}\text { 21st October } 2009 \\
\text { Autumn }\end{array}$ \\
\hline 1. & General suspension & $\mathrm{mg} \mathrm{O}_{2} \mathrm{dm}^{-3}$ & 17.4 (II) & 20.1 (II) & 18.3 (II) \\
\hline 2. & $\mathrm{pH}$ & - & $7.68(\mathrm{I})$ & $7.70(\mathrm{I})$ & 7.58 (I) \\
\hline 3. & COD-Mn & $\mathrm{mg} \mathrm{O}_{2} \mathrm{dm}^{-3}$ & 4.6 (II) & 5.3 (II) & 4.8 (II) \\
\hline 4. & $\mathrm{BOD}_{5}$ & $\mathrm{mg} \mathrm{O}_{2} \mathrm{dm}^{-3}$ & 2.7 (II) & 2.6 (II) & 2.3 (II) \\
\hline 5. & $\mathrm{O}_{2 \text { diss. }}$ & $\mathrm{mg} \mathrm{O}_{2} \mathrm{dm}^{-3}$ & 7.7 (I) & 7.6 (I) & $8.2(\mathrm{I})$ \\
\hline 6. & $\mathrm{NO}_{3}^{-}$ & $\mathrm{mg} \mathrm{N} \mathrm{dm}^{-3}$ & $0.85(\mathrm{I})$ & $0.96(\mathrm{I})$ & $0.53(\mathrm{I})$ \\
\hline 7. & $\mathrm{NO}_{2}^{-}$ & $\mathrm{mg} \mathrm{N} \mathrm{dm} \mathrm{m}^{-3}$ & 0.051 (I) & 0.063 (II) & 0.045 (II) \\
\hline 8. & $\mathrm{NH}_{4}^{+}$ & $\mathrm{mg} \mathrm{N} \mathrm{dm}{ }^{-3}$ & 0.63 (II) & 0.79 (II) & 0.64 (II) \\
\hline 9. & $\mathrm{PO}_{4}^{3-}$ diss. & $\mathrm{mg} \mathrm{PO}_{4} \mathrm{dm}^{-3}$ & 0.25 (II) & 0.32 (II) & 0.22 (II) \\
\hline 10. & $\mathrm{P}_{\text {Total }}$ & $\mathrm{mg} \mathrm{P} \mathrm{dm}^{-3}$ & 0.22 (II) & 0.29 (II) & 0.24 (II) \\
\hline \multicolumn{6}{|c|}{ Year 2010} \\
\hline S.No. & Water quality indices & Units & $\begin{array}{c}\text { 21st April } 2010 \\
\text { Spring } \\
\end{array}$ & $\begin{array}{c}\text { 14th July } 2010 \\
\text { Summer }\end{array}$ & $\begin{array}{c}\text { 20th October } 2010 \\
\text { Autumn }\end{array}$ \\
\hline 1. & General suspension & $\mathrm{mg} \mathrm{O}_{2} \mathrm{dm}^{-3}$ & 18.1 (II) & 19.5 (II) & $16.4(\mathrm{II})$ \\
\hline 2. & $\mathrm{pH}$ & - & $7.72(\mathrm{I})$ & $7.68(\mathrm{I})$ & 7.70 (I) \\
\hline 3. & COD-Mn & $\mathrm{mg} \mathrm{O}_{2} \mathrm{dm}^{-3}$ & 4.3 (II) & $5.1 \mathrm{II})$ & 4.8 (II) \\
\hline 4. & $\mathrm{BOD}_{5}$ & $\mathrm{mg} \mathrm{O}_{2} \mathrm{dm}^{-3}$ & 2.4 (II) & 2.6 (II) & 2.2 (II) \\
\hline 5. & $\mathrm{O}_{2 \text { diss. }}$ & $\mathrm{mg} \mathrm{O}_{2} \mathrm{dm}^{-3}$ & $8.1(\mathrm{I})$ & 7.4 (I) & 7.8 (I) \\
\hline 6. & $\mathrm{NO}_{3}^{-}$ & $\mathrm{mg} \mathrm{N} \mathrm{dm}{ }^{-3}$ & $0.91(\mathrm{I})$ & $1.38(\mathrm{I})$ & $1.38(\mathrm{I})$ \\
\hline 7. & $\mathrm{NO}_{2}^{-}$ & $\mathrm{mg} \mathrm{N} \mathrm{dm} \mathrm{dm}^{-3}$ & 0.043 (I) & 0.074 (II) & 0.052 (II) \\
\hline 8. & $\mathrm{NH}_{4}^{+}$ & $\mathrm{mg} \mathrm{N} \mathrm{dm}{ }^{-3}$ & 0.67 (II) & 0.79 (II) & 0.58 (II) \\
\hline 9. & $\mathrm{PO}_{4}^{3-}$ diss. & $\mathrm{mg} \mathrm{PO} \mathrm{Pdm}^{-3}$ & 0.26 (II) & 0.34 (II) & 0.21 (II) \\
\hline 10. & $\mathrm{P}_{\text {Total }}$ & $\mathrm{mg} \mathrm{P} \mathrm{dm}{ }^{-3}$ & 0.30 (II) & 0.33 (II) & 0.27 (II) \\
\hline \multicolumn{6}{|c|}{ Year 2011} \\
\hline S.No. & Water quality indices & Units & $\begin{array}{c}\text { 20April } 2011 \\
\text { Spring }\end{array}$ & $\begin{array}{l}\text { 20th July } 2011 \\
\text { Summer } \\
\end{array}$ & $\begin{array}{c}\text { 19th October } 2011 \\
\text { Autumn }\end{array}$ \\
\hline 1. & General suspension & $\mathrm{mg} \mathrm{O}_{2} \mathrm{dm}^{-3}$ & 17.3 (II) & 19.7 (II) & 16.4 (II) \\
\hline 2. & $\mathrm{pH}$ & - & $7.72(\mathrm{I})$ & 7.69 (I) & $7.82(\mathrm{I})$ \\
\hline 3. & COD-Mn & $\mathrm{mg} \mathrm{O}_{2} \mathrm{dm}^{-3}$ & 4.6 (II) & 5.3 (II) & 4.8 (II) \\
\hline 4. & $\mathrm{BOD}_{5}$ & $\mathrm{mg} \mathrm{O}_{2} \mathrm{dm}^{-3}$ & 2.1 (II) & 2.7 (II) & 2.4 (II) \\
\hline 5. & $\mathrm{O}_{2 \text { diss. }}$ & $\mathrm{mg} \mathrm{O}_{2} \mathrm{dm}^{-3}$ & 8.2 (I) & 7.5 (I) & 7.9 (I) \\
\hline 6. & $\mathrm{NO}_{3}^{-}$ & $\mathrm{mg} \mathrm{N} \mathrm{dm}{ }^{-3}$ & $0.74(\mathrm{I})$ & $0.93(\mathrm{I})$ & 0.69 (I) \\
\hline 7. & $\mathrm{NO}_{2}^{-}$ & $\mathrm{mg} \mathrm{N} \mathrm{dm}^{-3}$ & 0.053 (II) & 0.078 (II) & 0.061 (II) \\
\hline 8. & $\mathrm{NH}_{4}^{+}$ & $\mathrm{mg} \mathrm{N} \mathrm{dm}{ }^{-3}$ & 0.61 (II) & 0.79 (II) & $0.58(\mathrm{I})$ \\
\hline 9. & $\mathrm{PO}_{4}^{3-}$ diss. & $\mathrm{mg} \mathrm{PO}_{4} \mathrm{dm}^{-3}$ & 0.23 (II) & 0.34 (II) & 0.25 (II) \\
\hline 10. & $\mathrm{P}_{\text {Total }}$ & $\mathrm{mg} \mathrm{P} \mathrm{dm}^{-3}$ & 0.21 (II) & 0.29 (II) & 0.24 (II) \\
\hline \multicolumn{6}{|c|}{ Year 2012} \\
\hline S.No. & Water quality indices & Units & $\begin{array}{c}\text { 18th April } 2012 \\
\text { Spring } \\
\end{array}$ & $\begin{array}{c}\text { 18th July } 2012 \\
\text { Summer }\end{array}$ & $\begin{array}{c}\text { 27th September } 2012 \\
\text { Autumn }\end{array}$ \\
\hline 1. & General suspension & $\mathrm{mg} \mathrm{O}_{2} \mathrm{dm}^{-3}$ & 16.7 (II) & 21.4 (II) & 20.2 (II) \\
\hline 2. & $\mathrm{pH}$ & - & $7.63(\mathrm{I})$ & $7.70(\mathrm{I})$ & 7.74 (I) \\
\hline 3. & COD-Mn & $\mathrm{mg} \mathrm{O}_{2} \mathrm{dm}^{-3}$ & 4.2 (II) & 5.3 (II) & 4.5 (II) \\
\hline 4. & $\mathrm{BOD}_{5}$ & $\mathrm{mg} \mathrm{O}_{2} \mathrm{dm}^{-3}$ & 2.4 (II) & 2.6 (II) & 2.2 (II) \\
\hline 5. & $\mathrm{O}_{2 \text { diss. }}$ & $\mathrm{mg} \mathrm{O}_{2} \mathrm{dm}^{-3}$ & $8.3(\mathrm{I})$ & $7.3(\mathrm{I})$ & 7.8 (I) \\
\hline 6. & $\mathrm{NO}_{3}^{-}$ & $\mathrm{mg} \mathrm{N} \mathrm{dm}{ }^{-3}$ & $0.62(\mathrm{I})$ & 0.79 (I) & $0.84(\mathrm{I})$ \\
\hline 7. & $\mathrm{NO}_{2}^{-}$ & $\mathrm{mg} \mathrm{N} \mathrm{dm}{ }^{-3}$ & 0.049 (II) & 0.085 (II) & 0.072 (II) \\
\hline 8. & $\mathrm{NH}_{4}^{+}$ & $\mathrm{mg} \mathrm{N} \mathrm{dm}^{-3}$ & 0.53 (II) & 0.75 (II) & $0.63(\mathrm{I})$ \\
\hline 9. & $\mathrm{PO}_{4}^{3-}$ diss. & $\mathrm{mg} \mathrm{PO}_{4} \mathrm{dm}^{-3}$ & 0.26 (II) & 0.29 (II) & 0.20 (II) \\
\hline 10. & $\mathrm{P}_{\text {Total }}$ & $\mathrm{mg} \mathrm{P} \mathrm{dm}{ }^{-3}$ & 0.30 (II) & 0.36 (II) & 0.22 (II) \\
\hline
\end{tabular}




\begin{tabular}{|c|c|c|c|c|c|}
\hline \multicolumn{6}{|c|}{$\begin{array}{c}\text { RESULTS OF THE QUALITY OF SURFACE WATER OF PRZYLEG LAKE (SPRING, SUMMER AND AUTUMN 2008-2012) } \\
\text { ALONG WITH THE CLASSIFICATION VALUES OF INDICATORS ACCORDING TO THE CRITERIA OF } \\
\text { THE EUROPEAN UNION WATER FRAMEWORK DIRECTIVE (2000/60/EC) }\end{array}$} \\
\hline \multicolumn{6}{|c|}{ Year 2008} \\
\hline S.No. & Water quality indices & Units & $\begin{array}{l}\text { 17th April } 2008 \\
\text { Spring }\end{array}$ & $\begin{array}{c}\text { 24th July } 2008 \\
\text { Summer }\end{array}$ & $\begin{array}{c}\text { 15th October2008 } \\
\text { Autumn }\end{array}$ \\
\hline 1. & General suspension & $\mathrm{mg} \mathrm{O}_{2} \mathrm{dm}^{-3}$ & $26.5(\mathrm{III})$ & 29.3 (III) & $26.7(\mathrm{III})$ \\
\hline 2. & $\mathrm{pH}$ & - & 7.64 (I) & $7.72(\mathrm{I})$ & $7.81(\mathrm{I})$ \\
\hline 3. & COD-Mn & $\mathrm{mg} \mathrm{O}_{2} \mathrm{dm}^{-3}$ & 7.3 (III) & 8.6 (III) & 8.3 (III) \\
\hline 4. & $\mathrm{BOD}_{5}$ & $\mathrm{mg} \mathrm{O}_{2} \mathrm{dm}^{-3}$ & 4.7 (III) & 5.3 (III) & 5.1 (III) \\
\hline 5. & $\mathrm{O}_{2 \text { diss. }}$ & $\mathrm{mg} \mathrm{O}_{2} \mathrm{dm}^{-3}$ & $8.4(\mathrm{I})$ & $7.4(\mathrm{I})$ & $7.3(\mathrm{I})$ \\
\hline 6. & $\mathrm{NO}_{3}^{-}$ & $\mathrm{mg} \mathrm{N} \mathrm{dm}^{-3}$ & $0.67(\mathrm{I})$ & $0.72(\mathrm{I})$ & 0.59 (I) \\
\hline 7. & $\mathrm{NO}_{2}^{-}$ & $\mathrm{mg} \mathrm{N} \mathrm{dm}^{-3}$ & 0.063 (II) & 0.084 (II) & 0.069 (II) \\
\hline 8. & $\mathrm{NH}_{4}^{+}$ & $\mathrm{mg} \mathrm{N} \mathrm{dm}^{-3}$ & 0.73 (II) & 0.87 (II) & 0.52 (II) \\
\hline 9. & $\mathrm{PO}_{4}^{3-}$ diss. & $\mathrm{mg} \mathrm{PO}_{4} \mathrm{dm}^{-3}$ & 0.53 (III) & 0.62 (III) & 0.47 (III) \\
\hline 10. & $\mathrm{P}_{\text {Total }}$ & $\mathrm{mg} \mathrm{P} \mathrm{dm}{ }^{-3}$ & 0.47 (III) & 0.57 (III) & 0.42 (III) \\
\hline \multicolumn{6}{|c|}{ Year 2009} \\
\hline S.No. & Water quality indices & Units & $\begin{array}{l}\text { 15th April } 2009 \\
\text { Spring }\end{array}$ & $\begin{array}{c}\text { 22nd July } 2009 \\
\text { Summer }\end{array}$ & $\begin{array}{c}\text { 21st October } 2009 \\
\text { Autumn }\end{array}$ \\
\hline 1. & General suspension & $\mathrm{mg} \mathrm{O}_{2} \mathrm{dm}^{-3}$ & 27.5 (III) & 29.1 (III) & $28.4(\mathrm{III})$ \\
\hline 2. & $\mathrm{pH}$ & - & $7.61(\mathrm{I})$ & $7.85(\mathrm{I})$ & $7.58(\mathrm{I})$ \\
\hline 3. & COD-Mn & $\mathrm{mg} \mathrm{O}_{2} \mathrm{dm}^{-3}$ & 7.1 (III) & 9.8 (III) & 8.2 (III) \\
\hline 4. & $\mathrm{BOD}_{5}$ & $\mathrm{mg} \mathrm{O}_{2} \mathrm{dm}^{-3}$ & 5.1 (III) & 5.7 (III) & 4.9 (III) \\
\hline 5. & $\mathrm{O}_{2 \text { diss. }}$ & $\mathrm{mg} \mathrm{O}_{2} \mathrm{dm}^{-3}$ & $9.5(\mathrm{I})$ & 7.7 (I) & $8.4(\mathrm{I})$ \\
\hline 6. & $\mathrm{NO}_{3}^{-}$ & $\mathrm{mg} \mathrm{N} \mathrm{dm}{ }^{-3}$ & $0.83(\mathrm{I})$ & $0.97(\mathrm{I})$ & $0.72(\mathrm{I})$ \\
\hline 7. & $\mathrm{NO}_{2}^{-}$ & $\mathrm{mg} \mathrm{N} \mathrm{dm}{ }^{-3}$ & 0.068 (II) & 0.083 (II) & 0.056 (II) \\
\hline 8. & $\mathrm{NH}_{4}^{+}$ & $\mathrm{mg} \mathrm{N} \mathrm{dm}^{-3}$ & 0.68 (II) & 0.82 (II) & 0.59 (II) \\
\hline 9. & $\mathrm{PO}_{4}^{3-}$ diss. & $\mathrm{mg} \mathrm{PO}_{4} \mathrm{dm}^{-3}$ & 0.45 (III) & 0.64 (III) & 0.52 (III) \\
\hline 10. & $\mathrm{P}_{\text {Total }}$ & $\mathrm{mg} \mathrm{P} \mathrm{dm}{ }^{-3}$ & 0.49 (III) & 0.57 (III) & 0.45 (III) \\
\hline \multicolumn{6}{|c|}{ Year 2010} \\
\hline S.No. & Water quality indices & Units & $\begin{array}{l}\text { 21st April } 2010 \\
\text { Spring }\end{array}$ & $\begin{array}{l}\text { 14th July } 2010 \\
\text { Summer }\end{array}$ & $\begin{array}{c}\text { 20th October } 2010 \\
\text { Autumn }\end{array}$ \\
\hline 1. & General suspension & $\mathrm{mg} \mathrm{O}_{2} \mathrm{dm}^{-3}$ & 26.4 (III) & 28.9 (III) & 26.5 (III) \\
\hline 2. & $\mathrm{pH}$ & - & $7.62(\mathrm{I})$ & $7.73(\mathrm{I})$ & $7.58(\mathrm{I})$ \\
\hline 3. & COD-Mn & $\mathrm{mg} \mathrm{O}_{2} \mathrm{dm}^{-3}$ & 7.3 (III) & 8.3 (III) & 7.8 (III) \\
\hline 4. & $\mathrm{BOD}_{5}$ & $\mathrm{mg} \mathrm{O}_{2} \mathrm{dm}^{-3}$ & 4.2 (III) & 6.5 (III) & 5.4 (III) \\
\hline 5. & $\mathrm{O}_{2 \text { diss. }}$ & $\mathrm{mg} \mathrm{O}_{2} \mathrm{dm}^{-3}$ & $8.3(\mathrm{I})$ & 7.9 (I) & 8.9 (I) \\
\hline 6. & $\mathrm{NO}_{3}^{-}$ & $\mathrm{mg} \mathrm{N} \mathrm{dm}{ }^{-3}$ & $0.82(\mathrm{I})$ & $0.92(\mathrm{I})$ & $0.76(\mathrm{I})$ \\
\hline 7. & $\mathrm{NO}_{2}^{-}$ & $\mathrm{mg} \mathrm{N} \mathrm{dm}{ }^{-3}$ & 0.077 (II) & 0.083 (II) & 0.074 (II) \\
\hline 8. & $\mathrm{NH}_{4}^{+}$ & $\mathrm{mg} \mathrm{N} \mathrm{dm}^{-3}$ & 0.74 (II) & 0.82 (II) & 0.70 (II) \\
\hline 9. & $\mathrm{PO}_{4}^{3-}$ diss. & $\mathrm{mg} \mathrm{PO}_{4} \mathrm{dm}^{-3}$ & 0.53 (III) & 0.59 (III) & 0.46 (III) \\
\hline 10. & $\mathrm{P}_{\text {Total }}$ & $\mathrm{mg} \mathrm{P} \mathrm{dm}{ }^{-3}$ & 0.46 (III) & 0.63 (III) & 0.49 (III) \\
\hline \multicolumn{6}{|c|}{ Year 2011} \\
\hline S.No. & Water quality indices & Units & $\begin{array}{l}\text { 20th April } 2011 \\
\text { Spring }\end{array}$ & $\begin{array}{l}\text { 20th July } 2011 \\
\text { Summer }\end{array}$ & $\begin{array}{c}\text { 19th October } 2011 \\
\text { Autumn }\end{array}$ \\
\hline 1. & General suspension & $\mathrm{mg} \mathrm{O}_{2} \mathrm{dm}^{-3}$ & 27.4 (III) & 31.6 (III) & 30.2 (III) \\
\hline 2. & $\mathrm{pH}$ & - & $7.62(\mathrm{I})$ & $7.80(\mathrm{I})$ & 7.74 (I) \\
\hline 3. & COD-Mn & $\mathrm{mg} \mathrm{O}_{2} \mathrm{dm}^{-3}$ & 7.6 (III) & 8.4 (III) & 8.9 (III) \\
\hline 4. & $\mathrm{BOD}_{5}$ & $\mathrm{mg} \mathrm{O}_{2} \mathrm{dm}^{-3}$ & 4.7 (III) & 5.2 (III) & 4.4 (III) \\
\hline 5. & $\mathrm{O}_{2 \text { diss. }}$ & $\mathrm{mg} \mathrm{O}_{2} \mathrm{dm}^{-3}$ & $8.3(\mathrm{I})$ & $7.7(\mathrm{I})$ & $8.6(\mathrm{I})$ \\
\hline 6. & $\mathrm{NO}_{3}^{-}$ & $\mathrm{mg} \mathrm{N} \mathrm{dm}^{-3}$ & $0.82(\mathrm{I})$ & $0.68(\mathrm{I})$ & $0.92(\mathrm{I})$ \\
\hline 7. & $\mathrm{NO}_{2}^{-}$ & $\mathrm{mg} \mathrm{N} \mathrm{dm}{ }^{-3}$ & 0.063 (II) & 0.078 (II) & 0.069 (II) \\
\hline 8. & $\mathrm{NH}_{4}^{+}$ & $\mathrm{mg} \mathrm{N} \mathrm{dm}^{-3}$ & 0.56 (II) & 0.87 (II) & 0.63 (II) \\
\hline 9. & $\mathrm{PO}_{4}^{3-}$ diss. & $\mathrm{mg} \mathrm{PO}_{4} \mathrm{dm}^{-3}$ & 0.53 (III) & 0.68 (III) & 0.44 (III) \\
\hline 10. & $\mathrm{P}_{\text {Total }}$ & $\mathrm{mg} \mathrm{P} \mathrm{dm}{ }^{-3}$ & 0.49 (III) & 0.53 (III) & 0.46 (III) \\
\hline \multicolumn{6}{|c|}{ Year 2012} \\
\hline S.No. & Water quality indices & Units & $\begin{array}{c}\text { 18th April } 2012 \\
\text { Spring }\end{array}$ & $\begin{array}{c}\text { 18th July } 2012 \\
\text { Summer }\end{array}$ & $\begin{array}{c}\text { 27th September } 2012 \\
\text { Autumn }\end{array}$ \\
\hline 1. & General suspension & $\mathrm{mg} \mathrm{O}_{2} \mathrm{dm}^{-3}$ & 24.0 (III) & 19.0 (III) & 26.3 (III) \\
\hline 2. & $\mathrm{pH}$ & - & $7.71(\mathrm{I})$ & $7.70(\mathrm{I})$ & $7.65(\mathrm{I})$ \\
\hline 3. & COD-Mn & $\mathrm{mg} \mathrm{O}_{2} \mathrm{dm}^{-3}$ & 8.8 (III) & 9.5 (III) & 9.3 (III) \\
\hline 4. & $\mathrm{BOD}_{5}$ & $\mathrm{mg} \mathrm{O}_{2} \mathrm{dm}^{-3}$ & 4.3 (III) & 5.5 (III) & 5.6 (III) \\
\hline 5. & $\mathrm{O}_{2 \text { diss. }}$ & $\mathrm{mg} \mathrm{O}_{2} \mathrm{dm}^{-3}$ & 7.9 (I) & $7.3(\mathrm{I})$ & $8.6(\mathrm{I})$ \\
\hline 6. & $\mathrm{NO}_{3}^{-}$ & $\mathrm{mg} \mathrm{N} \mathrm{dm}{ }^{-3}$ & $0.79(\mathrm{I})$ & $0.60(\mathrm{I})$ & $0.86(\mathrm{I})$ \\
\hline 7. & $\mathrm{NO}_{2}^{-}$ & $\mathrm{mg} \mathrm{N} \mathrm{dm}^{-3}$ & 0.052 (II) & 0.095 (II) & 0.074 (II) \\
\hline 8. & $\mathrm{NH}_{4}^{+}$ & $\mathrm{mg} \mathrm{N} \mathrm{dm}{ }^{-3}$ & 0.73 (II) & 0.62 (II) & 0.59 (II) \\
\hline 9. & $\mathrm{PO}_{4}^{3-}$ diss. & $\mathrm{mg} \mathrm{PO}_{4} \mathrm{dm}^{-3}$ & 0.48 (III) & 0.59 (III) & 0.45 (III) \\
\hline 10. & $\mathrm{P}_{\text {Total }}$ & $\mathrm{mg} \mathrm{P} \mathrm{dm}^{-3}$ & 0.43 (III) & 0.67 (III) & 0.49 (III) \\
\hline
\end{tabular}




\begin{tabular}{|c|c|c|c|c|c|}
\hline \multicolumn{6}{|c|}{$\begin{array}{c}\text { RESULTS OF THE QUALITY OF SURFACE WATER OF CHLOP LAKE (SPRING, SUMMER AND AUTUMN } 2008 \text { - 2012) } \\
\text { ALONG WITH THE CLASSIFICATION VALUES OF INDICATORS ACCORDING TO THE CRITERIA OF } \\
\text { THE EUROPEAN UNION WATER FRAMEWORK DIRECTIVE (2000/60/EC) }\end{array}$} \\
\hline \multicolumn{6}{|c|}{ Year 2008} \\
\hline S.No. & Water quality indices & Units & $\begin{array}{l}\text { 17th April } 2008 \\
\text { Spring }\end{array}$ & $\begin{array}{l}\text { 24th July } 2008 \\
\text { Summer }\end{array}$ & $\begin{array}{c}\text { 15th October } 2008 \\
\text { Autumn }\end{array}$ \\
\hline 1. & General suspension & $\mathrm{mg} \mathrm{O}_{2} \mathrm{dm}^{-3}$ & 22.7 (II) & 24.2 (II) & 18.7 (II) \\
\hline 2. & $\mathrm{pH}$ & - & $7.62(\mathrm{I})$ & 7.70 (I) & 7.73 (I) \\
\hline 3. & COD-Mn & $\mathrm{mg} \mathrm{O}_{2} \mathrm{dm}^{-3}$ & 3.9 (II) & 5.3 (II) & 4.8 (II) \\
\hline 4. & $\mathrm{BOD}_{5}$ & $\mathrm{mg} \mathrm{O}_{2} \mathrm{dm}^{-3}$ & 2.6 (II) & 3.7 (III) & 2.5 (II) \\
\hline 5. & $\mathrm{O}_{2 \text { diss. }}$ & $\mathrm{mg} \mathrm{O}_{2} \mathrm{dm}^{-3}$ & $8.1(\mathrm{I})$ & 7.7 (I) & $7.9(\mathrm{I})$ \\
\hline 6. & $\mathrm{NO}_{3}-$ & $\mathrm{mg} \mathrm{N} \mathrm{dm}^{-3}$ & $0.92(\mathrm{I})$ & $0.83(\mathrm{I})$ & $0.75(\mathrm{I})$ \\
\hline 7. & $\mathrm{NO}_{2}^{-}$ & $\mathrm{mg} \mathrm{N} \mathrm{dm}-3$ & 0.037 (II) & 0.038 (II) & 0.032 (II) \\
\hline 8. & $\mathrm{NH}_{4}^{+}$ & $\mathrm{mg} \mathrm{N} \mathrm{dm}^{-3}$ & 0.62 (II) & 0.74 (II) & 0.59 (II) \\
\hline 9. & $\mathrm{PO}_{4}{ }^{3-}$ diss. & $\mathrm{mg} \mathrm{PO}_{4} \mathrm{dm}^{-3}$ & 0.25 (II) & 0.36 (II) & 0.22 (II) \\
\hline 10. & $\mathrm{P}_{\text {tot. }}$ & $\mathrm{mg} \mathrm{P} \mathrm{dm}^{-3}$ & 0.23 (II) & 0.36 (II) & 0.24 (II) \\
\hline \multicolumn{6}{|c|}{ Year 2009} \\
\hline S.No. & Water quality indices & Units & $\begin{array}{c}\text { 15th April } 2009 \\
\text { Spring }\end{array}$ & $\begin{array}{c}\text { 22nd July } 2009 \\
\text { Summer }\end{array}$ & $\begin{array}{c}\text { 21st October } 2009 \\
\text { Autumn }\end{array}$ \\
\hline 1. & General suspension & $\mathrm{mg} \mathrm{O}_{2} \mathrm{dm}^{-3}$ & 18.4 (II) & 21.6 (II) & 17.2 (II) \\
\hline 2. & $\mathrm{pH}$ & - & $7.68(\mathrm{I})$ & $7.53(\mathrm{I})$ & $7.62(\mathrm{I})$ \\
\hline 3. & COD-Mn & $\mathrm{mg} \mathrm{O}_{2} \mathrm{dm}^{-3}$ & 4.7 (II) & 5.1 (II) & 3.6 (II) \\
\hline 4. & $\mathrm{BOD}_{5}$ & $\mathrm{mg} \mathrm{O}_{2} \mathrm{dm}^{-3}$ & 2.7 (II) & 4.5 (III) & 2.6 (II) \\
\hline 5. & $\mathrm{O}_{2 \text { diss. }}$ & $\mathrm{mg} \mathrm{O}_{2} \mathrm{dm}^{-3}$ & 8.3 (I) & $7.6(\mathrm{I})$ & 8.7 (I) \\
\hline 6. & $\mathrm{NO}_{3}{ }^{-}$ & $\mathrm{mg} \mathrm{N} \mathrm{dm}{ }^{-3}$ & $0.78(\mathrm{I})$ & $0.81(\mathrm{I})$ & $0.72(\mathrm{I})$ \\
\hline 7. & $\mathrm{NO}_{2}^{-}$ & $\mathrm{mg} \mathrm{N} \mathrm{dm} \mathrm{m}^{-3}$ & 0.048 (II) & 0.072 (II) & 0.064 (II) \\
\hline 8. & $\mathrm{NH}_{4}^{+}$ & $\mathrm{mg} \mathrm{N} \mathrm{dm}{ }^{-3}$ & 0.71 (II) & 0.85 (II) & 0.63 (II) \\
\hline 9. & $\mathrm{PO}_{4}{ }^{3-}$ diss. & $\mathrm{mg} \mathrm{PO}_{4} \mathrm{dm}^{-3}$ & 0.23 (II) & 0.34 (II) & 0.25 (II) \\
\hline 10. & $\mathrm{P}_{\text {Total }}$ & $\mathrm{mg} \mathrm{P} \mathrm{dm}{ }^{-3}$ & 0.26 (II) & 0.29 (II) & 0.22 (II) \\
\hline \multicolumn{6}{|c|}{ Year 2010} \\
\hline S.No. & Water quality indices & Units & $\begin{array}{l}\text { 21st April } 2010 \\
\text { Spring }\end{array}$ & $\begin{array}{l}\text { 14th July } 2010 \\
\text { Summer }\end{array}$ & $\begin{array}{c}\text { 20th October } 2010 \\
\text { Autumn }\end{array}$ \\
\hline 1. & General suspension & $\mathrm{mg} \mathrm{O}_{2} \mathrm{dm}^{-3}$ & 17.4 (II) & 19.3 (II) & $16.8(\mathrm{II})$ \\
\hline 2. & $\mathrm{pH}$ & - & 7.67 (I) & $7.71(\mathrm{I})$ & $7.84(\mathrm{I})$ \\
\hline 3. & COD-Mn & $\mathrm{mg} \mathrm{O} \mathrm{dm}^{-3}$ & 3.3 (II) & 4.5 (II) & 3.1 (II) \\
\hline 4. & $\mathrm{BOD}_{5}$ & $\mathrm{mg} \mathrm{O}_{2} \mathrm{dm}^{-3}$ & 2.2 (II) & 4.7 (III) & 2.5 (II) \\
\hline 5. & $\mathrm{O}_{2 \text { diss. }}$ & $\mathrm{mg} \mathrm{O}_{2} \mathrm{dm}^{-3}$ & 8.3 (I) & 7.3 (I) & 7.9 (I) \\
\hline 6. & $\mathrm{NO}_{3}{ }^{-}$ & $\mathrm{mg} \mathrm{N} \mathrm{dm}{ }^{-3}$ & $0.86(\mathrm{I})$ & $0.92(\mathrm{I})$ & $0.52(\mathrm{I})$ \\
\hline 7. & $\mathrm{NO}_{2}^{-}$ & $\mathrm{mg} \mathrm{N} \mathrm{dm} \mathrm{dm}^{-3}$ & 0.052 (I) & 0.075 (II) & 0.059 (II) \\
\hline 8. & $\mathrm{NH}_{4}^{+}$ & $\mathrm{mg} \mathrm{N} \mathrm{dm}{ }^{-3}$ & 0.56 (II) & 0.72 (II) & 0.83 (II) \\
\hline 9. & $\mathrm{PO}_{4}^{3-}$ diss. & $\mathrm{mg} \mathrm{PO}_{4} \mathrm{dm}^{-3}$ & 0.28 (II) & 0.34 (II) & 0.21 (II) \\
\hline 10. & $\mathrm{P}_{\text {Total }}$ & $\mathrm{mg} \mathrm{P} \mathrm{dm}{ }^{-3}$ & 0.23 (II) & 0.28 (II) & 0.25 (II) \\
\hline \multicolumn{6}{|c|}{ Year 2011} \\
\hline S.No. & Water quality indices & Units & $\begin{array}{l}\text { 20th April } 2011 \\
\text { Spring }\end{array}$ & $\begin{array}{l}\text { 20th July } 2011 \\
\text { Summer }\end{array}$ & $\begin{array}{c}\text { 19th October } 2011 \\
\text { Autumn }\end{array}$ \\
\hline 1. & General suspension & $\mathrm{mg} \mathrm{O}_{2} \mathrm{dm}^{-3}$ & 18.0 (II) & 21.5 (II) & $16.9(\mathrm{II})$ \\
\hline 2. & $\mathrm{pH}$ & - & $7.72(\mathrm{I})$ & 7.75 (I) & $7.83(\mathrm{I})$ \\
\hline 3. & COD-Mn & $\mathrm{mg} \mathrm{O}_{2} \mathrm{dm}^{-3}$ & 3.6 (II) & 4.4 (II) & 3.8 (II) \\
\hline 4. & $\mathrm{BOD}_{5}$ & $\mathrm{mg} \mathrm{O}_{2} \mathrm{dm}^{-3}$ & 2.3 (II) & 2.7 (III) & 2.2 (II) \\
\hline 5. & $\mathrm{O}_{2 \text { diss. }}$ & $\mathrm{mg} \mathrm{O}_{2} \mathrm{dm}^{-3}$ & $8.2(\mathrm{I})$ & 7.4 (I) & 7.9 (I) \\
\hline 6. & $\mathrm{NO}_{3}^{-}$ & $\mathrm{mg} \mathrm{N} \mathrm{dm}{ }^{-3}$ & 0.68 (I) & $0.84(\mathrm{I})$ & $0.95(\mathrm{I})$ \\
\hline 7. & $\mathrm{NO}_{2}^{-}$ & $\mathrm{mg} \mathrm{N} \mathrm{dm}{ }^{-3}$ & 0.073 (I) & 0.092 (II) & 0.068 (II) \\
\hline 8. & $\mathrm{NH}_{4}^{+}$ & $\mathrm{mg} \mathrm{N} \mathrm{dm}{ }^{-3}$ & 0.85 (II) & 0.87 (II) & 0.57 (II) \\
\hline 9. & $\mathrm{PO}_{4}{ }^{3-}$ diss. & $\mathrm{mg} \mathrm{PO}_{4} \mathrm{dm}^{-3}$ & 0.21 (II) & 0.25 (II) & 0.20 (II) \\
\hline 10. & $\mathrm{P}_{\text {Total }}$ & $\mathrm{mg} \mathrm{P} \mathrm{dm}{ }^{-3}$ & 0.25 (II) & 0.27 (II) & 0.24 (II) \\
\hline \multicolumn{6}{|c|}{ Year 2012} \\
\hline S.No. & Water quality indices & Units & $\begin{array}{c}\text { 18th April } 2012 \\
\text { Spring }\end{array}$ & $\begin{array}{c}\text { 18th July } 2012 \\
\text { Summer }\end{array}$ & $\begin{array}{c}\text { 27th September } 2012 \\
\text { Autumn }\end{array}$ \\
\hline 1. & General suspension & $\mathrm{mg} \mathrm{O}_{2} \mathrm{dm}^{-3}$ & 20.5 (II) & 23.7 (II) & 18.6 (II) \\
\hline 2. & $\mathrm{pH}$ & - & $7.64(\mathrm{I})$ & $7.71(\mathrm{I})$ & 7.75 (I) \\
\hline 3. & COD-Mn & $\mathrm{mg} \mathrm{O}_{2} \mathrm{dm}^{-3}$ & 4.1 (II) & 5.3 (II) & 4.2 (II) \\
\hline 4. & $\mathrm{BOD}_{5}$ & $\mathrm{mg} \mathrm{O}_{2} \mathrm{dm}^{-3}$ & 2.1 (II) & 2.6 (II) & 2.4 (II) \\
\hline 5. & $\mathrm{O}_{2 \text { diss. }}$ & $\mathrm{mg} \mathrm{O}_{2} \mathrm{dm}^{-3}$ & 8.1 (I) & $7.3(\mathrm{I})$ & $8.6(\mathrm{I})$ \\
\hline 6. & $\mathrm{NO}_{3}^{-}$ & $\mathrm{mg} \mathrm{N} \mathrm{dm}^{-3}$ & $0.79(\mathrm{I})$ & 0.98 (I) & $0.86(\mathrm{I})$ \\
\hline 7. & $\mathrm{NO}_{2}^{-}$ & $\mathrm{mg} \mathrm{N} \mathrm{dm}{ }^{-3}$ & $0.074(\mathrm{I})$ & 0.079 (II) & 0.062 (II) \\
\hline 8. & $\mathrm{NH}_{4}^{+}$ & $\mathrm{mg} \mathrm{N} \mathrm{dm}{ }^{-3}$ & 0.74 (II) & 0.77 (II) & 0.62 (II) \\
\hline 9. & $\mathrm{PO}_{4}{ }^{3-}$ diss. & $\mathrm{mg} \mathrm{PO} \mathrm{dm}^{-3}$ & 0.23 (II) & 0.26 (II) & 0.24 (II) \\
\hline 10. & $\mathrm{P}_{\text {Total }}$ & $\mathrm{mg} \mathrm{P} \mathrm{dm}^{-3}$ & 0.21 (II) & 0.35 (II) & 0.29 (II) \\
\hline
\end{tabular}



ALONG WITH THE CLASSIFICATION VALUES OF INDICATORS ACCORDING TO THE CRITERIA OF THE EUROPEAN UNION WATER FRAMEWORK DIRECTIVE (2000/60/EC)

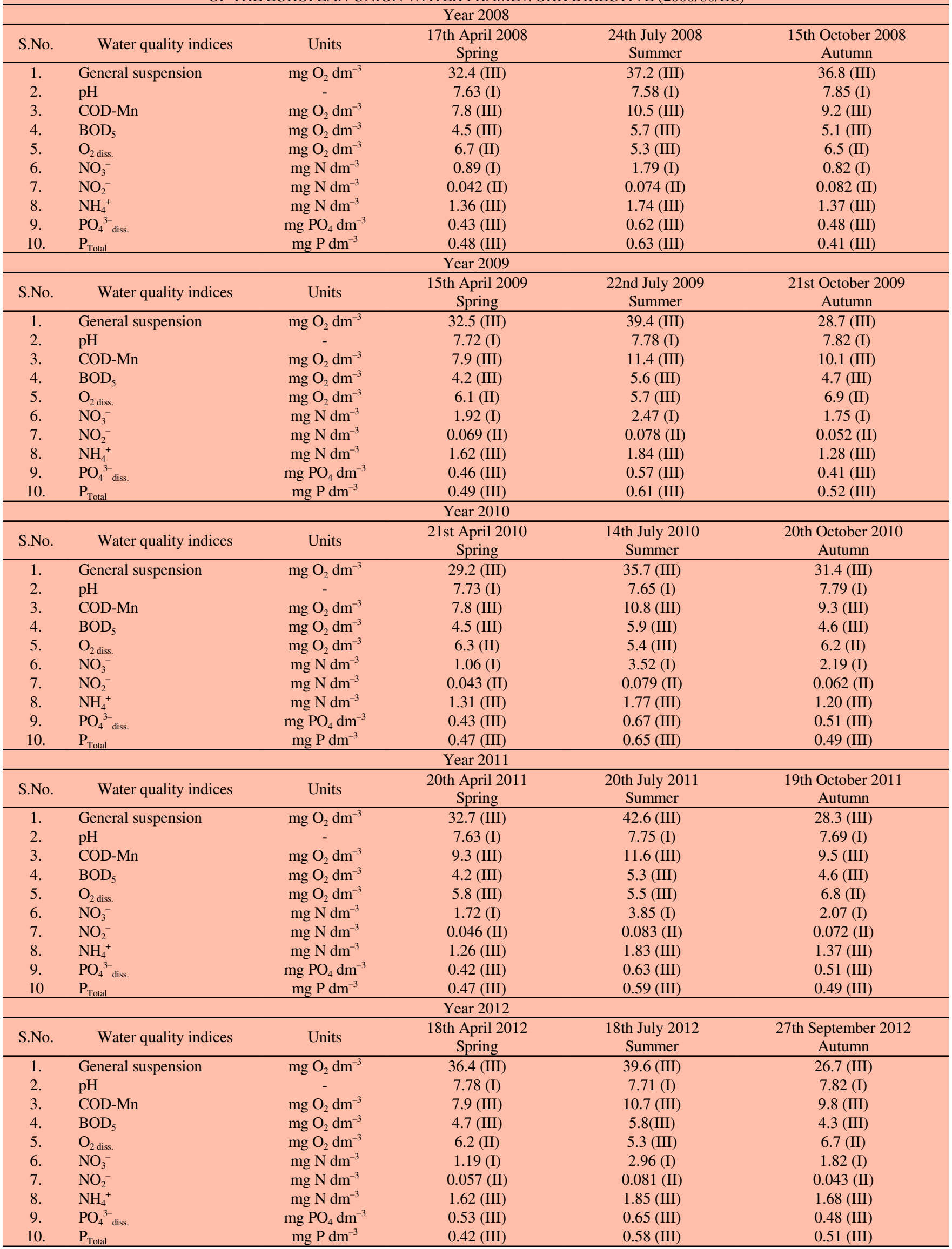




\begin{tabular}{|c|c|c|c|c|c|}
\hline \multicolumn{6}{|c|}{$\begin{array}{c}\text { RESULTS OF THE QUALITY OF SURFACE WATER OF WIELGIE LAKE (SPRING, SUMMER AND AUTUMN 2008-2012) } \\
\text { ALONG WITH THE CLASSIFICATION VALUES OF INDICATORS ACCORDING TO THE CRITERIA } \\
\text { OF THE EUROPEAN UNION WATER FRAMEWORK DIRECTIVE (2000/60/EC) }\end{array}$} \\
\hline \multicolumn{6}{|c|}{ Year 2008} \\
\hline S.No. & Water quality indices & Units & $\begin{array}{c}\text { 17th April } 2008 \\
\text { Spring }\end{array}$ & $\begin{array}{l}\text { 24th July } 2008 \\
\text { Summer }\end{array}$ & $\begin{array}{c}\text { 15th October } 2008 \\
\text { Autumn }\end{array}$ \\
\hline w & General suspension & $\mathrm{mg} \mathrm{O}_{2} \mathrm{dm}^{-3}$ & 29.3 (III) & 43.5 (III) & 32.8 (III) \\
\hline 2. & $\mathrm{pH}$ & - & $7.64(\mathrm{I})$ & $7.71(\mathrm{I})$ & $7.79(\mathrm{I})$ \\
\hline 3. & COD-Mn & $\mathrm{mg} \mathrm{O}_{2} \mathrm{dm}^{-3}$ & 7.8 (III) & 10.8 (III) & 9.3 (III) \\
\hline 4. & $\mathrm{BOD}_{5}$ & $\mathrm{mg} \mathrm{O}_{2} \mathrm{dm}^{-3}$ & 4.5 (III) & 5.3 (III) & 4.1 (III) \\
\hline 5. & $\mathrm{O}_{2 \text { diss. }}$ & $\mathrm{mg} \mathrm{O}_{2} \mathrm{dm}^{-3}$ & 5.8 (III) & 5.1 (III) & 6.3 (II) \\
\hline 6. & $\mathrm{NO}_{3}^{-}$ & $\mathrm{mg} \mathrm{N} \mathrm{dm}{ }^{-3}$ & $1.62(\mathrm{I})$ & 4.68 (I) & 2.41 (I) \\
\hline 7. & $\mathrm{NO}_{2}^{-}$ & $\mathrm{mg} \mathrm{N} \mathrm{dm}^{-3}$ & 0.051 (II) & 0.074 (II) & 0.057 (II) \\
\hline 8. & $\mathrm{NH}_{4}^{+}$ & $\mathrm{mg} \mathrm{N} \mathrm{dm}{ }^{-3}$ & 1.46 (III) & 1.72 (III) & 0.93 (III) \\
\hline 9. & $\mathrm{PO}_{4}^{3-}$ diss. & $\mathrm{mg} \mathrm{PO}_{4} \mathrm{dm}^{-3}$ & 0.49 (III) & 0.59 (III) & 0.45 (III) \\
\hline 10. & $\mathrm{P}_{\text {Total }}$ & $\mathrm{mg} \mathrm{P} \mathrm{dm}{ }^{-3}$ & 0.47 (III) & 0.63 (III) & 0.46 (III) \\
\hline \multicolumn{6}{|c|}{ Year 2009} \\
\hline S.No. & Water quality indices & Units & $\begin{array}{l}\text { 15th April } 2009 \\
\text { Spring }\end{array}$ & $\begin{array}{l}\text { 22nd July } 2009 \\
\text { Summer }\end{array}$ & $\begin{array}{c}\text { 21st October } 2009 \\
\text { Autumn }\end{array}$ \\
\hline 1. & General suspension & $\mathrm{mg} \mathrm{O}_{2} \mathrm{dm}^{-3}$ & 33.7 (III) & 46.3 (III) & $31.6(\mathrm{III})$ \\
\hline 2. & $\mathrm{pH}$ & - & $7.74(\mathrm{I})$ & 7.79 (I) & $7.82(\mathrm{I})$ \\
\hline 3. & COD-Mn & $\mathrm{mg} \mathrm{O}_{2} \mathrm{dm}^{-3}$ & 8.1 (III) & 11.6 (III) & 9.3 (III) \\
\hline 4. & $\mathrm{BOD}_{5}$ & $\mathrm{mg} \mathrm{O}_{2} \mathrm{dm}^{-3}$ & 4.5 (III) & 5.7 (III) & 4.3 (III) \\
\hline 5. & $\mathrm{O}_{2 \text { diss. }}$ & $\mathrm{mg} \mathrm{O}_{2} \mathrm{dm}^{-3}$ & 5.7 (III) & 5.3 (III) & 6.8 (II) \\
\hline 6. & $\mathrm{NO}_{3}^{-}$ & $\mathrm{mg} \mathrm{N} \mathrm{dm}^{-3}$ & $1.75(\mathrm{I})$ & $4.58(\mathrm{I})$ & $3.61(\mathrm{I})$ \\
\hline 7. & $\mathrm{NO}_{2}^{-}$ & $\mathrm{mg} \mathrm{N} \mathrm{dm}^{-3}$ & 0.052 (II) & 0.084 (II) & 0.061 (II) \\
\hline 8. & $\mathrm{NH}_{4}^{+}$ & $\mathrm{mg} \mathrm{N} \mathrm{dm}{ }^{-3}$ & 1.34 (III) & 1.79 (III) & 1.39 (III) \\
\hline 9. & $\mathrm{PO}_{4}^{3-}$ diss. & $\mathrm{mg} \mathrm{PO}_{4} \mathrm{dm}^{-3}$ & 0.57 (III) & 0.68 (III) & 0.48 (III) \\
\hline 10. & $\mathrm{P}_{\text {Total }}$ & $\mathrm{mg} \mathrm{P} \mathrm{dm}^{-3}$ & 0.46 (III) & 0.59 (III) & 0.53 (III) \\
\hline \multicolumn{6}{|c|}{ Year 2010} \\
\hline S.No. & Water quality indices & Units & $\begin{array}{l}\text { 21st April } 2010 \\
\text { Spring }\end{array}$ & $\begin{array}{l}\text { 14th July } 2010 \\
\text { Summer }\end{array}$ & $\begin{array}{c}\text { 20th October } 2010 \\
\text { Autumn }\end{array}$ \\
\hline 1. & General suspension & $\mathrm{mg} \mathrm{O}_{2} \mathrm{dm}^{-3}$ & 29.4 (III) & 45.8 (III) & $28.6(\mathrm{III})$ \\
\hline 2. & $\mathrm{pH}$ & - & $7.76(\mathrm{I})$ & $7.84(\mathrm{I})$ & $7.81(\mathrm{I})$ \\
\hline 3. & COD-Mn & $\mathrm{mg} \mathrm{O}_{2} \mathrm{dm}^{-3}$ & 8.3 (III) & 10.7 (III) & 9.3 (III) \\
\hline 4. & $\mathrm{BOD}_{5}$ & $\mathrm{mg} \mathrm{O}_{2} \mathrm{dm}^{-3}$ & 4.3 (III) & 5.4 (III) & 4.8 (III) \\
\hline 5. & $\mathrm{O}_{2 \text { diss. }}$ & $\mathrm{mg} \mathrm{O}_{2} \mathrm{dm}^{-3}$ & 5.4 (III) & 5.2 (III) & 6.5 (II) \\
\hline 6. & $\mathrm{NO}_{3}^{-}$ & $\mathrm{mg} \mathrm{N} \mathrm{dm}^{-3}$ & $2.02(\mathrm{I})$ & $4.71(\mathrm{I})$ & $2.83(\mathrm{I})$ \\
\hline 7. & $\mathrm{NO}_{2}^{-}$ & $\mathrm{mg} \mathrm{N} \mathrm{dm}^{-3}$ & 0.062 (II) & 0.086 (II) & 0.059 (II) \\
\hline 8. & $\mathrm{NH}_{4}^{+}$ & $\mathrm{mg} \mathrm{N} \mathrm{dm}{ }^{-3}$ & 1.42 (III) & 1.84 (III) & 1.48 (III) \\
\hline 9. & $\mathrm{PO}_{4}^{3-}$ diss. & $\mathrm{mg} \mathrm{PO}_{4} \mathrm{dm}^{-3}$ & 0.51 (III) & 0.58 (III) & 0.56 (III) \\
\hline 10. & $\mathrm{P}_{\text {Total }}$ & $\mathrm{mg} \mathrm{P} \mathrm{dm}{ }^{-3}$ & 0.43 (III) & 0.67 (III) & 0.47 (III) \\
\hline \multicolumn{6}{|c|}{ Year 2011} \\
\hline S.No. & Water quality indices & Units & $\begin{array}{l}\text { 20th April } 2011 \\
\text { Spring }\end{array}$ & $\begin{array}{l}\text { 20th July } 2011 \\
\text { Summer }\end{array}$ & $\begin{array}{c}\text { 19th October } 2011 \\
\text { Autumn }\end{array}$ \\
\hline 1. & General suspension & $\mathrm{mg} \mathrm{O}_{2} \mathrm{dm}^{-3}$ & 32.8 (III) & 43.7 (III) & 29.4 (III) \\
\hline 2. & $\mathrm{pH}$ & - & $7.78(\mathrm{I})$ & 7.67 (I) & $7.75(\mathrm{I})$ \\
\hline 3. & COD-Mn & $\mathrm{mg} \mathrm{O}_{2} \mathrm{dm}^{-3}$ & 8.3 (III) & 10.8 (III) & 9.5 (III) \\
\hline 4. & $\mathrm{BOD}_{5}$ & $\mathrm{mg} \mathrm{O}_{2} \mathrm{dm}^{-3}$ & 3.8 (III) & 5.3 (III) & 4.5 (III) \\
\hline 5. & $\mathrm{O}_{2 \text { diss. }}$ & $\mathrm{mg} \mathrm{O}_{2} \mathrm{dm}^{-3}$ & 5.7 (III) & 5.2 (III) & 6.2 (III) \\
\hline 6. & $\mathrm{NO}_{3}{ }^{-}$ & $\mathrm{mg} \mathrm{N} \mathrm{dm}^{-3}$ & $2.81(\mathrm{I})$ & 3.70 (I) & $1.93(\mathrm{I})$ \\
\hline 7. & $\mathrm{NO}_{2}^{-}$ & $\mathrm{mg} \mathrm{N} \mathrm{dm}{ }^{-3}$ & 0.061 (II) & 0.079 (II) & 0.057 (II) \\
\hline 8. & $\mathrm{NH}_{4}^{+}$ & $\mathrm{mg} \mathrm{N} \mathrm{dm}{ }^{-3}$ & 1.64 (III) & 1.87 (III) & 1.25 (III) \\
\hline 9. & $\mathrm{PO}_{4}^{3-}$ diss. & $\mathrm{mg} \mathrm{PO}_{4} \mathrm{dm}^{-3}$ & 0.45 (III) & 0.60 (III) & 0.57 (III) \\
\hline 10. & $\mathrm{P}_{\text {Total }}^{+ \text {aiss. }}$ & $\mathrm{mg} \mathrm{Pdm}{ }^{-3}$ & 0.48 (III) & 0.58 (III) & 0.41 (III) \\
\hline \multicolumn{6}{|c|}{ Year 2012} \\
\hline S.No. & Water quality indices & Units & $\begin{array}{l}\text { 18th April } 2012 \\
\text { Spring }\end{array}$ & $\begin{array}{l}\text { 18th July } 2012 \\
\text { Summer }\end{array}$ & $\begin{array}{c}\text { 27th September } 2012 \\
\text { Autumn }\end{array}$ \\
\hline 1. & General suspension & $\mathrm{mg} \mathrm{O}_{2} \mathrm{dm}^{-3}$ & 36.2 (III) & 46.3 (III) & 31.9 (III) \\
\hline 2. & $\mathrm{pH}$ & - & 7.64 (I) & $7.71(\mathrm{I})$ & $7.68(\mathrm{I})$ \\
\hline 3. & COD-Mn & $\mathrm{mg} \mathrm{O}_{2} \mathrm{dm}^{-3}$ & 7.2 (III) & 11.6 (III) & 10.2 (III) \\
\hline 4. & $\mathrm{BOD}_{5}$ & $\mathrm{mg} \mathrm{O}_{2} \mathrm{dm}^{-3}$ & 3.2 (III) & 5.9 (III) & 5.0 (III) \\
\hline 5. & $\mathrm{O}_{2 \text { diss. }}$ & $\mathrm{mg} \mathrm{O}_{2} \mathrm{dm}^{-3}$ & 5.8 (III) & 5.1 (III) & 6.4 (II) \\
\hline 6. & $\mathrm{NO}_{3}{ }^{-}$ & $\mathrm{mg} \mathrm{N} \mathrm{dm}^{-3}$ & $3.52(\mathrm{I})$ & $4.36(\mathrm{I})$ & $2.09(\mathrm{I})$ \\
\hline 7. & $\mathrm{NO}_{2}^{-}$ & $\mathrm{mg} \mathrm{N} \mathrm{dm}{ }^{-3}$ & 0.058 (II) & 0.084 (II) & 0.062 (II) \\
\hline 8. & $\mathrm{NH}_{4}^{+}$ & $\mathrm{mg} \mathrm{N} \mathrm{dm}{ }^{-3}$ & 1.27 (III) & 1.79 (III) & 1.14 (III) \\
\hline 9. & $\mathrm{PO}_{4}^{3-}$ diss. & $\mathrm{mg} \mathrm{PO}_{4} \mathrm{dm}^{-3}$ & 0.51 (III) & 0.63 (III) & 0.49 (III) \\
\hline 10. & $\mathrm{P}_{\text {Total }}$ diss. & $\mathrm{mg} \mathrm{P} \mathrm{dm}{ }^{-3}$ & 0.54 (III) & 0.62 (III) & 0.53 (III) \\
\hline
\end{tabular}


the form of gas dissolved in the water, $\mathrm{NH}_{4}{ }^{+}, \mathrm{NO}_{3}{ }^{-}$and $\mathrm{NO}_{2}^{-}$. In lakes, it is the main factor limiting the growth of organisms ${ }^{1-5,7,9-12,14,15,18,20-27,29,30}$.

The tests have demonstrated that the water quality in the lakes with regard to the tested indicators varied. By analyzing the average annual values, one can note that the $\mathrm{pH}, \mathrm{O}_{2 \text { diss }}$ and $\mathrm{NO}_{3}{ }^{-}$concentration showed a relatively small variation in all the investigated lakes. The total suspended solids in the lakes Barlineckie, Glebokie, Suche, Lubiszewko, Przyleg and Chlop, fell into the II class, while the lakes Lubie and Wielgie met the criteria of the III class.

The $\mathrm{P}_{\text {tot. }}$ concentrations in the surface layer of the lakes was little differentiated, reaching the levels appropriate for the II and III quality class according to the classification of the European Union Water Framework Directive. The total phosphorus concentration was $0.21-0.67 \mathrm{mg} \mathrm{dm}^{-3}$. The highest concentration of total phosphorus was recorded in Lakes Przyleg and Wielgie. The concentrations of $\mathrm{PO}_{4}{ }^{3-}$ diss in the tested lake waters varied more significantly-corresponding to water quality classes ranging from II through V. An upswing in the concentration of phosphorus compounds in a lake may indicate a decreased amount of oxygen in the benthic waters and changes in their redox status leading to releasing phosphorus compounds accumulated in the bed sediment $t^{1-5,7,9-12,14,15,18,20-27,29,30}$.

In the case of nitrogen compounds, nitrates and nitrites values for these indicators fell into the I and II class in all the surveyed lakes in accordance with the classification of the European Union Water Framework Directive. The indicator which proves high productivity of the lakes is the biochemical oxygen demand $\left(\mathrm{BOD}_{5}\right)$. The level of this indicator in the studied Lakes was at level II and III. The highest concentration of oxygen in the lake waters was found in the Lake Barlineckie (about $9.7 \mathrm{mg} \mathrm{O}_{2} \mathrm{dm}^{-3}$ ). In the remaining lakes oxygen levels were similar (still in I class).

\section{REFERENCES}

1. P. Daniszewski, Int. Lett. Chem. Phys. Astron., 1, 6 (2012).

2. P. Daniszewski, Int. Lett. Chem. Phys. Astron., 1, 13 (2012).

3. P. Daniszewski, Int. Lett. Chem. Phys. Astron., 2, 42 (2012).

4. P. Daniszewski, Int. Lett. Chem. Phys. Astron., 2, 35 (2012).
5. P. Daniszewski and R. Konieczny, Int. Lett. Chem. Phys. Astron., 4, 91 (2013).

6. A. Kownacki, H. Soszka, D. Kudelska and T. Flejtuch, Bioassessment of Polish rivers based on macroinvertebrates; In: 11th Magdeburg seminar on Waters In Central and Eastern Europe: Assessment, Protection, Manage-ment, W. Geller, et al. (eds.). Proceedings of the international conference, 18-22 October (2004).

7. N.L. Nemerow, Stream, Lake, Estuary, and Ocean Pollution, Van Nostrand Reinhold Company, New York, pp. 185-189 (1985).

8. G. Van Urk and B. de Vaate, Limnologie Aktuell. 1, 131 (1990).

9. E. Bajkiewicz-Grabowska, J. Hydrol. Sci., 8, 63 (1981).

10. E. Bécares, Limnetica, 25, 143 (2006).

11. P. Daniszewski, Int. Lett. Chem. Phys. Astron., 2, 46 (2012).

12. T. Fleituch, H. Soszka, D. Kudelska and A. Kownacki, Hydrobiol. Suppl. 141, 3225 (2002).

13. Z. Kajak, Hydrobiology-Limnology, Inland Water Ecosystems, PWN, Warsaw, pp. 355 (1998).

14. Z. Kajak, Ekol. Pol., 31, 495 (1983)

15. P. Psenner, B. Boström, M. Dinka, K. Pettersson, R. Pucsko and M. Sager, Arch. Hydrobiol. Beih. Ergebn. Limnol. 30, 83 (1988).

16. Z. Chudecki and L. Duda, Pol. Soil Sci., 4, 145 (1971).

17. Directive 2000/60/EC of the European Parliament and of the Council of 23 October 2000 Establishing a Framework for Community Action in the Field of Water Policy, Off. J. Eur. Commun. L 327, 22 December (2000).

18. F. Garcia-Criado, A. Tomé, F.J. Vega and C. Antolin, Hydrobiologia, 394, 209 (1999).

19. A. Kownacki and H. Soszka, Guidelines for the evaluation of the status of rivers on the basis of macroinvertebrates and for intakes of macroinvertebrate samples in lakes, Warsaw, p. 51 (2004).

20. A. Lelek, Can. Special Pub. Fisheries Aquatic Sci., 106, 469 (1989).

21. A. Mudroch, J.M. Azcue and P. Mudroch, Physico-chemical Analysis of Aquatic Sediments, Lewis Publishers, Boca Raton, New York, London, Tokyo (1997).

22. J. Kubiak, Acta Sci. Pol. Piscaria, 2, 141 (2003).

23. W. Lampert and U. Sommer, Ecology of Inland Waters. Scientific Publishing PWN, Warsaw pp. 415 (2001).

24. C. Richards, G.E. Host and J.W. Arthur, Freshwat. Biol., 29, 285 (1993).

25. M. Søndergaard, K.D. Wolter and W. Ripl, in eds.: M.R. Perow and A.J. Davy, Chemical Treatment of water and Sediments with Special Reference to lakes; In: Handbook of Ecological Restoration, Cambridge University Press, Cambridge, pp. 184-205 (2002).

26. J.F. Wright, D. Moss, P.D. Armitage and M.T. Furs, Freshwat. Biol., 14, 221 (1984).

27. B. Zdanowski, Ekol. Pol., 31, 287 (1983).

28. J. Janczak, Atlas Polish Lakes, pp. 92-95 (1996).

29. J. Trojanowski and J. Bruski, Baltic Coastal Zone, 4, 53 (2000).

30. A. Macioszczyk, Hydrochemistry, Ed. Geology, Warsaw, p. 475 (1987).

30. J. Trojanowski, J. Antonowicz, M. Król and J. Bruski, Ann. Polish Chem. Soc., 1, 131 (2001). 